\title{
Review
}

\section{Humor and Laughter May Influence Health IV. Humor and Immune Function}

\author{
Mary Payne Bennett ${ }^{1}$ and Cecile Lengacher ${ }^{2}$ \\ ${ }^{1}$ Western Kentucky University, School of Nursing and ${ }^{2}$ University of South Florida
}

This is the final article in a four part series reviewing the influence of humor and laughter on physiological and psychological well-being. This final article reviews the evidence for the effect of sense of humor, exposure to a humor stimulus and laughter on various immune system components, with a focus on the effects of laughter on natural killer cell cytotoxicity.

Keywords: Humor-Laughter-Immune Function-Psychoneuroimmunology

\section{Introduction}

This article is the conclusion of a multipart series reviewing the evidence currently available concerning the influence of humor on various health outcomes. The first article in this series discussed the basic background of research in this area including definitions and a review of theoretical underpinnings (1). The second article reviewed the literature concerning use of humor as a complementary therapy within various clinical samples, as well as evidence concerning how a sense of humor influences physiological and psychological well-being (2). The third article in this series examined the influence of the physiological response to humor (i.e. laughter) on selected health outcomes; including muscle tension, cardio-respiratory functioning and various physiological measures of stress (3). This final article reviews the evidence thus far to support the influence of sense of humor, exposure to humor and laughter on various immune system outcomes.

\section{Sense of Humor and Immune Function}

According to PNI theory and several recent studies, it has been postulated that laughter or sense of humor may

For reprints and all correspondence: Mary Payne Bennett, Western Kentucky University, School of Nursing, AC Room 113D, 1906 College Heights Blvd \# 11036, Bowling Green, KY 42101-1036, USA.

E-mail: mary.bennett@wku.edu affect health through moderation of stress chemicals and/ or immunoenhancement (4-7). In order to test this theory, Martin and Dobbin (8) used a sample of 40 college students to determine if sense of humor might act as a moderator of daily hassles on immune functioning (8). Hassles were measured by Daily Hassles Scale (DHS), while sense of humor was measured using the Situational Humor Response Questionnaire (SHRQ), the Metamessage Sensitivity Subscale (SHQ-MS), the Liking of Humor Subscale (SHQ-LH) and the Coping Humor Scale (CHS). Immune function was determined by salivary $\operatorname{IgA}(\mathrm{SIgA})$. SIgA was measured at two different times during the study, about one and a half months apart. While scores on the SHRQ and SIgA at time two were positively correlated $(P<0.05)$, all the other humor scores and SIgA measure correlations were not significant, and six out of eight correlations were even negative (non-significant). These results would appear to indicate that sense of humor has no direct effect on salivary immune functioning, or at most, a very modest effect. The authors concluded that sense of humor, while not directly related to SIgA, might act as a buffer or moderator of the effects of daily hassles on SIgA (8).

However, this supposition proposed by Martin and Dobbin (8) was not supported by their subsequent study that attempted to document the moderating effect of sense of humor on the relationship between exam stress and a variety of immune measures. Dobbin and colleagues $(9,10)$, examined the effect of three psychological variables (Type A, Locus of Control and Sense 
of Humor measured by CHS) on immune functioning following examination stress. The results indicated that, as expected, exam stress produced significant immune suppression as measured by decreases in $\mathrm{T}$-cell response to various mitogens, decreased IL-1beta levels $(P<0.01)$ and decreased interferon gamma levels $(P<0.05)$. However, the effect of exam stress was not modified by sense of humor or any of the measured psychological variables used. The authors concluded that the role of psychological traits as moderators of stress should be questioned $(9,10)$. While this conclusion appears too broad, considering the bulk of literature to support the role of other psychological factors as stress modifiers, it does call into question the ability of sense of humor (at least as measured by CHS) to moderate the effects of stress on physiological functioning.

\section{Exposure to Humor, Subsequent Laughter and Salivary IgA}

In order to determine if simply exposing people to a humorous situation can result in immunoenhancement, a few small studies have been conducted using humor videos. In a randomized crossover design, 10 college students viewed two videos, a humorous video and an instructional video (used as a control) (11). The results of exposure to a humorous video and sense of humor (measured by CHS) on SIgA levels were examined. Sense of humor scores on the CHS were positively correlated $(P<0.05)$ with SIgA levels before viewing the videos, demonstrating that subjects with greater sense of humor also had increased salivary IgA, but scores on the CHS were actually negatively correlated with increases in SIgA $(P<0.10)$ following the humorous video. An attempt to explain this confounding finding was suggested by the authors, in that as the subjects with higher humor scores had higher baseline SIgA before the film, SIgA may not have been able to increase as much from the intervention, due to a possible ceiling effect. But given the conflicting data obtained when examining the relationship between sense of humor and immune function, it may be that sense of humor does not have a significant effect upon this physiological outcome measure.

On the positive side, the findings did demonstrate that subjects had significantly increased SIgA levels $(P<0.025)$ after watching the humorous video (Richard Pryor Live), indicating that exposure to humor (and indirectly, subsequent laughter or other humor response) can increase one measure of immune function (SIgA levels). This supports the popular view that humor can be healthy, but obviously needs additional support before adoption by the scientific community.

Another publication documents results of three separate investigations of the effect of humor on SIgA, using college students as subjects (4). In all three studies ( $n=45, n=34, N=62)$, subjects' salivary IgA levels increased significantly after a brief humorous stimulus $(P<0.005)$. These results clearly support the immunoenhancing actions of exposure to a brief humorous stimulus on SIgA. Again, the influence of sense of humor on SIgA levels was less clear. In an attempt to overcome possible problems due to instrumentation, sense of humor was measured using both SHRQ and CHS. Contrary to Dillon's findings, in study number one, scores on the humor scales were unrelated to baseline SIgA, while scores on the CHS were positively related to increases in SIgA following the stimulus $(P<0.02)$. In study number 2 , no significant correlation was found between sense of humor and pre- or post-SIgA levels; and in study number 3 , scores on the SHRQ were only marginally related to increases in post-stimulus $\operatorname{SIgA}(P<0.10)$.

In summary, the effect of sense of humor on SIgA and its possible interaction with the humorous stimulus is not clearly supported. One investigation reported that sense of humor was negatively correlated with increases in SIgA following the humorous stimulus (11), two others found subjects with greater sense of humor scores had larger increases in SIgA following the humorous stimulus (6), and lastly one reported sense of humor was not significantly related to SIgA levels before or following a humorous stimulus (6). On the other hand, the effect of exposure to humor stimulus on salivary $\operatorname{IgA}$ was supported. However, it should be noted that the use of salivary IgA as a measure of immune function has been questioned by some authors $(12,13)$, due to variations in individual saliva flow rate and the use of stimulated and non-stimulated samples in some studies. In addition, the clinical significance of increased levels of salivary $\operatorname{IgA}$ is questionable. Therefore, this body of research should be viewed with some caution and needs replication using a more valid and reliable measure of immune function.

\section{Exposure to Humor, Subsequent Laughter and Natural Killer Cell Cytotoxicity}

Many PNI researchers are now using natural killer cell cytotoxicity as an indicator of immune system functioning. According to Schulz et al. (14), the natural killer cell assay is the immunoassay most likely to produce clear and replicable results in this type of research. Natural killer (NK) cells are lymphocytes which are classified as a non-B, non-T or null cells. NK cells are able to lyze tumor cell line in vitro, while leaving normal cells intact (15). This demonstrates the ability of NK cells to discriminate between normal and cancerous cells. Additional in vitro data has demonstrated that NK cells are capable of killing a wide variety of cancerous cells including leukemia, carcinomas, sarcomas and melanomas $(15,16)$. Lower levels of $\mathrm{NK}$ cell activity 
have been correlated with the spread of cancer, demonstrated by increased tumor cell metastasis in both humans and animals (17). In addition, the NK cell is a spontaneous killer, in that it does not have to be activated by tumor antigens, nor is it limited by major histocompatibility complex requirements (18). There is also some evidence that natural killer cell activity is important in the prevention of some types of viral illnesses, most notably those induced by herpes simplex type 1, Epstein Barr and influenza viruses $(19,20)$.

Cardiovascular patients in Japan demonstrated a relationship between various psychological measures and immune function. A positive correlation was noted between NK cell activity and scores on both the extraversion scale and a sense of humor scale. They also reported a negative correlation between NK activity and scores on the neuroticism scale. The authors stated that the results indicate an association between general positive feelings and higher levels of NK cell activity. However, it should be noted that this work was correlational and did not examine cause and effect (21).

A few researchers have attempted to document the influence of humor and/or humor response on NK cytotoxicity. With 22 breast cancer patients as the sample in a randomized crossover design (22), all subjects viewed both a humorous video and a distressing video. NK cell cytotoxicity and numbers of NK cells were used as outcome measures. Half of the subjects viewed the humorous video first, while the other half viewed the distressing video first. Blood samples were taken from an indwelling catheter before and after each video, at $30 \mathrm{~min}$ following the video, and $23 \mathrm{~h}$ following the first video (just prior to viewing the second video). Sense of humor was also included and measured using the Coping Humor Scale (CHS). Scores on the CHS were positively correlated with numbers of NK cells, which would indicate that subjects with higher coping humor scores also had more NK cells. However, contrary to what was expected, no significant changes were noted in NK numbers or cytotoxicity at the end of either video or during recovery.

This unpublished dissertation also included an unexpected finding that NK cytotoxicity was significantly decreased $23 \mathrm{~h}$ after viewing the humorous video and was significantly increased $23 \mathrm{~h}$ after viewing the distressing film. Wise suggested that this could be a rebound effect, from a change in NK cytotoxicity (in the expected direction) sometime in the preceding $22 \mathrm{~h}$. However, it should be noted that the $23 \mathrm{~h}$ post-stimulus test point was immediately prior to viewing the second video. Perhaps this result was related to anticipation of the upcoming video. This is a confounding factor that needs to be avoided in future humor research. In addition, as NK level is known to be reduced in patients with cancer, using cancer patients as the subjects in this investigational work may have lead to an additional confounding factor.
Another investigation concerning the effect of humor on NK cell activity used 10 healthy male subjects (5). The experimental group viewed a $60 \mathrm{~min}$ humorous video, while the control group watched a non-humorous video. Blood samples were drawn from an indwelling catheter at baseline, during the movie, and $30 \mathrm{~min}$ after the movie (recovery). The experimental group displayed significantly increased NK cell activity during and following the humor video, from baseline to recovery $(P<0.008)$, while the control group did not. However, this study involved a very small sample and was published in abstract form only.

In what is probably the most comprehensive report thus far, Berk (23) reports the results of a series of five separate studies. The results are from a total of 52 healthy males, who viewed a humor video for $1 \mathrm{~h}$. Blood samples were taken $10 \mathrm{~min}$ before, $30 \mathrm{~min}$ into, $30 \mathrm{~min}$ after and $12 \mathrm{~h}$ after the intervention. Increases were found in NK cell activity $(P<0.01)$ and immunoglobulins $\mathrm{G}(P<0.02)$, A $(P<0.01)$ and $\mathrm{M}(P<0.09)$, with several immunoglobulin effects lasting at least $12 \mathrm{~h}$. The authors conclude that modulation of neuroimmune parameters by laughter may have indications for use in health and wellness intervention programs as adjunct to other integrative medicine therapies (23).

Most studies that document a positive effect of laughter on NK activity have used all male samples. The use of all male subjects in studies using immune or endocrine-based outcomes simplifies the methodology, as it negates any possible effects that may occur due to female hormonal changes. However, it then becomes difficult to determine how a particular intervention may work in women. Because of this, a later study by our group used a sample of 33 healthy adult women to examine the effect of laughter on stress and NK cell activity (24). This controlled study was designed to test the effect of humor on NK function, and to determine if sense of humor (measured by two different instruments) or laughter (measured by the Humor Response ScaleHRS) is related to change in stress NK activity when subjects are exposed to humor. Subjects were randomly assigned to view either a humor video or a neutral control video, with pre and post measures of stress and natural killer cell activity measured in all subjects.

Watching a humor video decreased stress for subjects in the humor group, as compared to those in the distraction group $(P<0.01)$. The effect of humor was apparently mediated by humor response, as amount of mirthful laughter correlated with the decreased stress seen in subjects who viewed the humor video $(P<0.01)$. Humor response also played a role in change in NK cell activity following the video. Subjects who scored less than 25 on the Humor Response Scale (indicating laughter) had increased immune function compared with their own preintervention immune function $(P<0.05)$, and compared to those participants who viewed the humor video but 
scored below 25 on the Humor Response Scale $(P<0.05)$. This finding indicated that only the subjects who laughed out loud during the humor video had significantly increased immune function following the intervention. Persons who just smiled or did not have observable responses to the humor video did not have positive changes in immune function. This finding is demonstrated in Fig. 1, indicating a significant relationship between the amount of laughter and change in immune function for persons in the humor group.

Similar to findings from other studies, there was no connection between sense of humor (as measured Situational Humor Response Scale and the Multidimensional

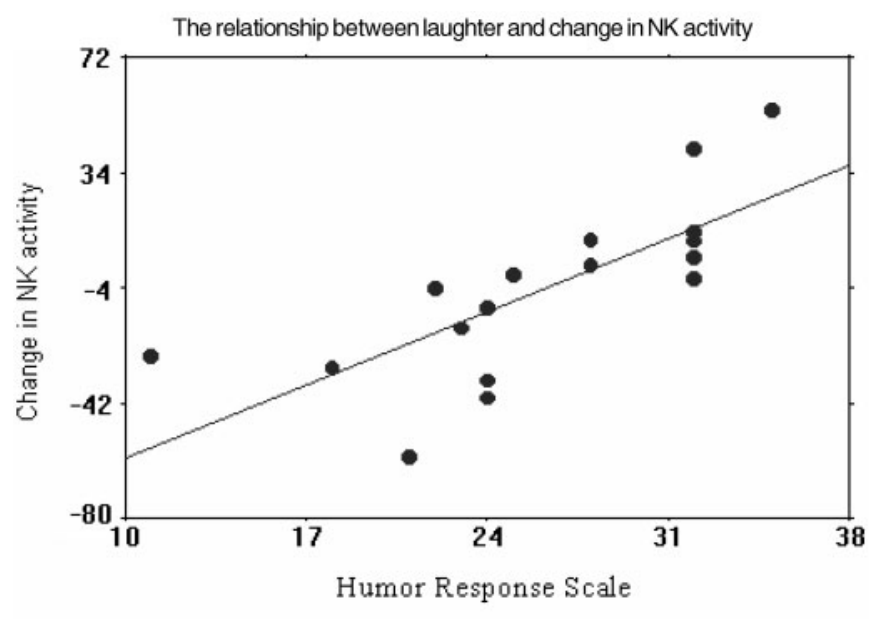

$r=0.774 P=0.001$

Figure 1. Plot represents the relationship between change in NK cell functioning and subject humor response (24).
Humor Scale) and change in any of the study outcomes. Sense of humor was not related to change in stress, change in NK activity or even observed laughter in response to the humor stimulus. This finding supports earlier work (8-10) that suggest that sense of humor (as measured by currently available instruments) does not appear to play a significant role in the physiological response to a humor stimulus. Findings from this study support that the act of laughing is correlated to changes in stress and immune function following exposure to a humor stimulus. Laughing can apparently reduce stress and improve NK cell activity, at least temporarily. As low NK cell activity is linked to decreased disease resistance and increased morbidity in persons with cancer and HIV disease, the authors conclude that laughter may be a useful cognitive-behavioral intervention for use in these patients. As an interesting side note, the differentiation in the effects of smiling versus laughing out loud was noted in another study of laughter, which examined the effect of smiling, laughing and howling on mood changes. It was found that howling did not substantially improve mood but both smiling and laughing did. Moreover, laughter seemed to boost positive affect more than just smiling (25). Additional support for the effect of laughter on various physiological outcomes is briefly summarized in Table 1.

\section{Discussion of Sense of Humor, Response to a Humor Stimulus and NK Cell Cytotoxicity}

Empirical evidence concerning the effect of sense of humor on NK cell function is conflicting; with one study

Table 1. Brief overview of outcomes using a humorous stimulus

\begin{tabular}{|c|c|}
\hline Health Outcome & Results \\
\hline Muscle relaxation & Periods of intense laughter are followed by relaxed muscle tone $(26,27)$. \\
\hline $\begin{array}{l}\text { Urinary epinephrine and norepinephrine } \\
\text { levels }\end{array}$ & $\begin{array}{l}\text { Hormonal measures of sympathetic nervous system activation increased during a humorous } \\
\text { video (28). }\end{array}$ \\
\hline Galvanic skin response and blood pressure & $\begin{array}{l}\text { A humorous stimulus lead to increased galvanic skin response, but stable blood pressure } \\
\text { indicating that while humor appears to involve activation of the sympathetic nervous } \\
\text { system (SNS), it also acts to buffer some of the actions of the SNS on blood pressure (29). }\end{array}$ \\
\hline Changes in heart and respiratory rate & Laughter leads to increased heart rate, respiratory rate and oxygen consumption (30). \\
\hline Relaxation as measured by Biofeedback & $\begin{array}{l}\text { College students who scored high on internal locus of control had increased relaxation } \\
\text { following exposure to a humorous stimulus (32). }\end{array}$ \\
\hline Various hormonal measures & $\begin{array}{l}\text { Berk (5). Serum cortisol, growth hormone and plasma dopac all decreased during the humorous } \\
\text { stimulus. No significant change was found in serum prolactin, beta endorphins, epinephrine } \\
\text { or norepinephrine. }\end{array}$ \\
\hline
\end{tabular}


finding that sense of humor was related to higher baseline levels of NK activity (23), while a second using an intervention design did not find any relationship between sense of humor and NK activity (24). One researcher did report that sense of humor, as measured by psychological self report instruments, is related to increased numbers of NK cells in the periphery (18), but this work needs replication in a larger sample. The overall impression obtained from the studies conducted thus far indicates that sense of humor has a minor role, if any, in changes in physiological outcomes when subjects are exposed to a humorous stimulus. However, it should be acknowledged that this finding may be due to inadequate methods currently available to measure sense of humor.

As for the effect of exposure to a humorous stimulus and subsequent laughter on measures of immune function, a few small studies demonstrate an increase in SIgA levels following exposure to a humorous video, but the clinical benefits of increasing SIgA levels are somewhat uncertain. Three out of four publications that report the effect of a humorous stimulus and/or laughter on NK cell activity indicate that exposure to a humorous stimulus or the act of laughter can increase NK cell activity, at least temporarily. However, it is not known if the increases demonstrated in these studies are of a significant value to have a clinical benefit, as long term studies looking at health outcomes have yet to be conducted. Therefore, additional research in this area is certainly indicated.

\section{Humor Research Methodology and Implications for Future Research}

As can been seen from the research reviewed in this article, humor researchers have used different conceptualizations of humor and different methodologies to examine the effect of humor, making it difficult to summarize this body of research. In some analyses, sense of humor is measured with a number of self-report instruments and then correlated with various health outcomes. The problem with this is that the various measures of sense of humor are subject to social desirability. Most people like to believe that they have a good or above average sense of humor, and thus this variable is difficult to capture using self-report instruments. Experimental design is also used to determine the effect of a humorous stimulus on health outcomes. However, what one person feels is a humorous movie or situation, another person may not view as being humorous. Therefore, while these studies may determine the outcome of viewing a supposedly humorous video in a given set of participants, there is no guarantee that the results obtained are, in fact, due to humor or laughter. In order to determine the effectiveness of humor or laughter, some objective measure of humor response is needed.
Unfortunately, most publications thus far have not included measurement of humor response.

As previous research indicates, some subjects who are exposed to a humorous stimulus do not laugh. In addition, what one person views as a humorous stimulus, another person may not find funny. This has been addressed in earlier work by allowing subjects a choice of different videos, etc., but it is still possible for a person to choose a video and still not find it funny, or at least not funny enough to produce laughter. Humor response is known to be affected by environmental conditions, and it can be difficult for subjects to relax and laugh at a humor video when experimental conditions require blood draws for laboratory testing. To help control for this, it is critical that future humor research includes some measure of subject response to the humorous stimulus. Videotaping subject responses and then measuring these responses with observer rating scales such the Humor Response Scale can help document the effect of individual humor responses to the stimulus.

Another concern is that of control groups. While experiments using a humorous stimulus should include some type of neutral stimulus to control for factors such as the passage of time, experimental conditions and the relaxation that might be associated with watching an interesting video of some type, many of the earlier studies did not include this type of control. It is also difficult to select a truly 'neutral stimulus', in that what one person may feel is a neutral stimulus, someone else may find amusing, etc. And to more completely control for other possible mechanisms which might affect health outcomes, Martin (33) has suggested the need for at least two additional control groups. A negative control group, which would watch a video intended to produce emotions such as fear or sadness, to control for the possible effects of 'general emotional arousal' and a positive control group who would view a video intended to produce positive emotions such as joy or happiness, but not necessarily laughter, to control for the effect of positive emotions.

Of course, all of these additional control groups add to the complexity of the design and analysis, and would require some measure of the emotions supposedly evoked by videos, to be sure that a subject who viewed the negative video actually experienced negative emotional arousal, etc. As can be seen, research design in this area can be quite challenging. Clearly, more groundwork is required to determine the best methods of testing and documenting psychological and physiological outcomes of humor in various populations. The next step is to incorporate the prior methodological work into future humor research design and investigate the phenomena in larger samples of healthy subjects. In addition, small clinical pilot studies may be used to investigate the possibly different effects of humor in different clinical populations. 


\section{Conclusions}

Research results concerning humor and healing are thus far rather tentative, and more work is needed before broad claims can be made concerning an effect of humor upon health outcomes. While in a frequently cited case study, Cousins (34) attributed his cure from ankylosing spondylitis to complementary therapies including laughter, empirical research supporting this type of response is currently not available. In fact, as can be seen from the above review, documentation of the effects of humor on various health related outcomes in healthy populations is still in the infancy stages, and research documenting benefits in a clinical population (such as persons with cancer) is yet to be established.

\section{References}

1. Bennett MP, Lengacher CA. Humor and laughter may influence health. I. History and background. Evid Based Complement Alternat Med 2006;3:61-3.

2. Bennett MP, Lengacher CA. Humor and laughter may influence health. II Complementary therapies and humor in a clinical population. Evid Based Complement Alternat Med 2006;3:187-90.

3. Bennett MP, Lengacher CA. Humor and laughter may influence health. Laughter and health outcomes. Evid Based Complement Alternat Med 2007; doi: 10.1093/ecam/nem041[Epub ahead of print].

4. Berk L, Tan S, Nehlsen-Cannarella S, Napier B, Lewis J, Lee J, et al. Humor associated laughter decreases cortisol and increases spontaneous lymphocyte blastogenesis. Clin Res 1988;36:435A.

5. Berk L, Tan S, Fry W, Napier B, Lee J, Hubbard R, et al. Neuroendocrine and stress hormone changes during mirthful laughter. Am J Med Sci 1989;298:391-6.

6. Lefcourt H, Davidson-Katz K, Kueneman K. Humor and immunesystem functioning. Humor: Int J Humor Res 1990;3:305-21.

7. Morreall J. Humor and work. Humor: Int J Humor Res 1991:4:359-73.

8. Martin R, Dobbin J. Sense of humor, hassles, and immunoglobulin A: evidence for a stress-moderating effect of humor. Int $J$ Psychiatr Med 1988;18:93-105.

9. Dobbin J. Individual Differences in the Appraisal of Stress and the Immunologic Consequences: Psychological Moderation of Lymphocyte Activation and Cytokine Production. Ontario: University of Western Ontario, 1990.

10. Dobbin J, Harth M, McCain G, Martin R. Cytokine production and lymphocyte transformation during stress. Brain Behav Immun 1991:5:339-48.

11. Dillon K, Minchoff B, Baker K. Positive emotional states and enhancement of the immune system. Int $J$ Psychiatr Med 1985;15:13-8.

12. Mouton C, Fillion L, Tawadros E, Tessier R. Salivary IgA is a weak stress marker. Ann Behav Med 1989;15:179-85.

13. Stone A, Cox D, Valdimarsdottir H. Neale J. Secretory IgA as a measure of immunocompetence. J Human Stress 1987;13:136-40.

14. Schulz R, Visintainer P, Williamson GP. Psychiatric and physical morbidity effects of care giving. J Gerontol 1990;45:181-91.
15. Herberman R, Ortaldo J. Natural killer cells: their role in defenses against disease. Science 1981;214:24-30.

16. Hanna N. In vivo activities of NK cells against primary and metastatic tumors in experimental animals. In: Lotzova E, Herberman R (eds). Immunobiology of Natural Killer Cells. Boca Raton, FLorida: CRC, 1986.

17. Hanna N, Barton R. Definitive evidence that natural killer (NK) cells inhibit experimental tumor metastasis in vivo. Immunology 1981;127:1754-8.

18. Locke S, Kraus L. Modulation of natural killer cell activity by life stress and coping ability. In: Levy S (ed). Biological Mediators of Behavior and Disease: Neoplasia. New York: Elsevier, 1982, 3-28.

19. Bonneau R, Sheriadan J, Feng N, Glaser R. Stress-induced effects on cell-mediated innate and adaptive memory components of the murine immune response to herpes simplex virus infection. Brain, Behav Immun 1991;5:274-95.

20. Esterling B, Antoni M, Schneiderman N, Carver C. Psychosocial modulation of antibody to Epstein-Barr viral capsid antigen and human herpesvirus type-6 in HIV-1-infected and at-risk gay men. Psychosom Med 1992;54:354-71.

21. Ishihara S, Nohara R, Makita S, Imai M, Kubo S, Hashimoto T. Immune function and psychological factors in patients with coronary heart disease. Jpn Circ J 1999;63:704-9.

22. Wise B. Comparison of immune response to mirth and to distress in women at risk for recurrent breast cancer. Diss Abstr Int 1989;49:2918.

23. Berk L, Felten D, Tan S, Bittman B, Westengard J. Modulation of neuroimmune parameters during the eustress of humorassociated mirthful laughter. Altern Ther HealthMed 2001;7:62-72; 74-76.

24. Bennett M, Zeller J, Rosenberg L, McCann J. The effect of mirthful laughter on stress and natural killer cell activity. Altern Ther HealthMed 2003;9:38-43.

25. Neuhoff C, Schaefer C. Effects of laughing, smiling, and howling on mood. Psychol Rep 2002;91:1079-80.

26. Paskind J. Effects of laughter on muscle tone. Arch Neurol Psychiarty 1932;28:623-8.

27. Overeem S, Taal W, Ocal Gezici E, Lammers G, Van Dijk J. Is motor inhibition during laughter due to emotional or respiratory influences? Psychophysiology 2004;41:254-8.

28. Levi L. The urinary output of adrenalin and noradrenalin during pleasant and unpleasant emotional states. Psychosom Medicine 1965;27:403-419.

29. Averill J. Autonomic response patterns during sadness and mirth. Psychophysiology 1969;5:399-414.

30. Fry W, Savin W. Mirthful laughter and blood pressure. Humor: Int J Humor Res 1988;1:49-62.

31. Yovetich N, Dale A, Hudak M. Benefits of humor in reduction of threat-induced anxiety. Psychol Rep 1990;66:51-8.

32. Prerost F. Presentation of humor and facilitation of a relaxation response among internal and external scores on Rotter's scale. Psycholo Rep 1993;72:1248-50.

33. Martin R. Humor, laughter, and physical health: methodological issues and research findings. Psychol Bull 2001;127:504-19.

34. Cousins N. Anatomy of an illness as perceived by the patient. Toronto: Bantam, 1979.

Received July 25, 2007; accepted July 26, 2007 


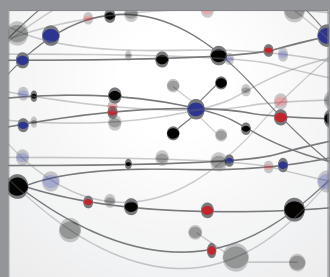

The Scientific World Journal
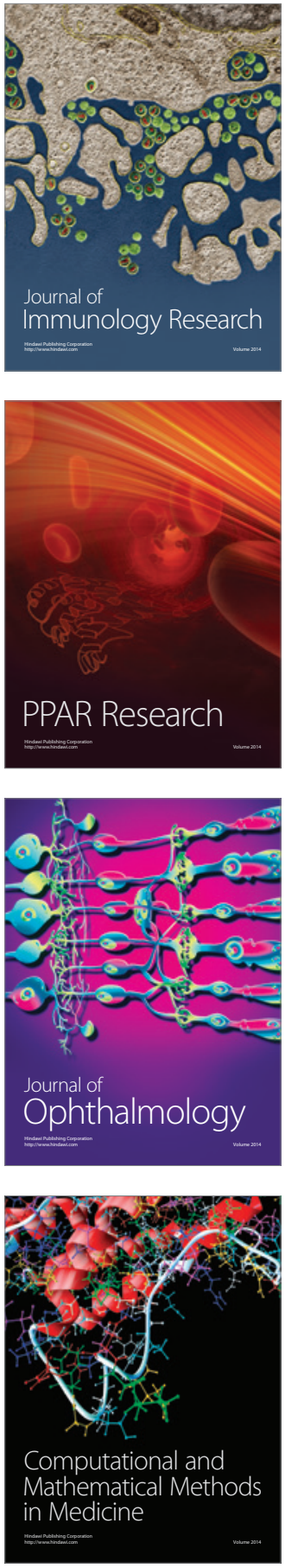

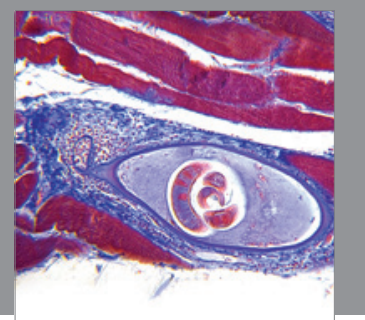

Gastroenterology

Research and Practice
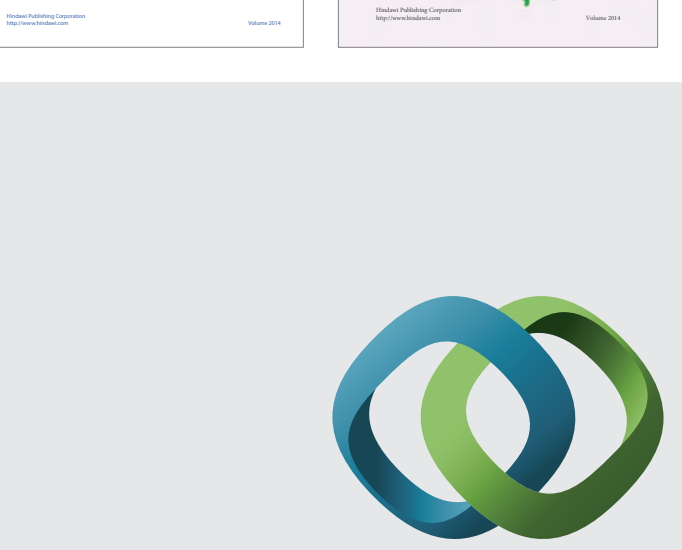

\section{Hindawi}

Submit your manuscripts at

http://www.hindawi.com
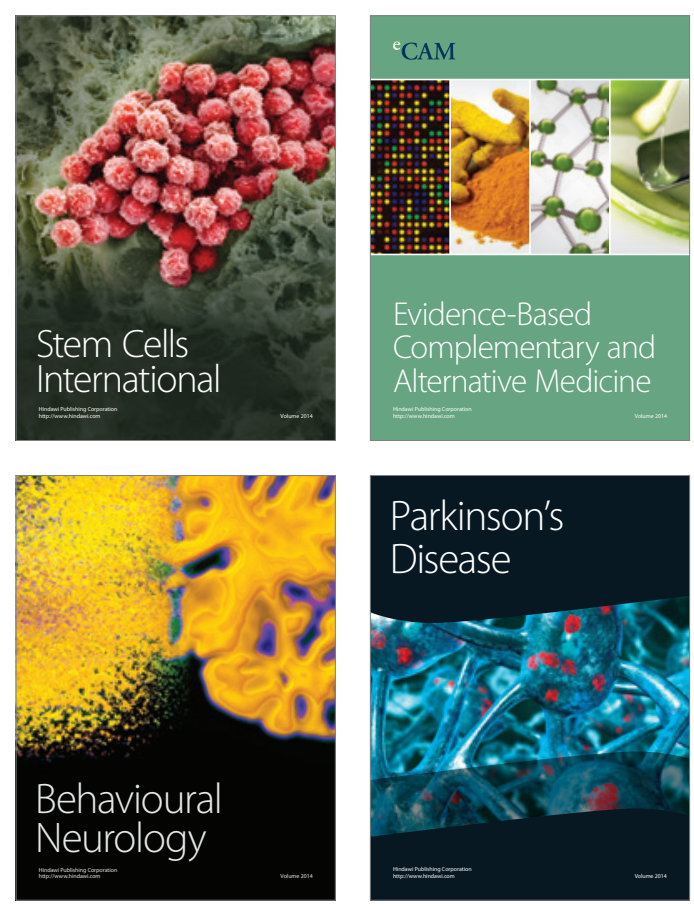

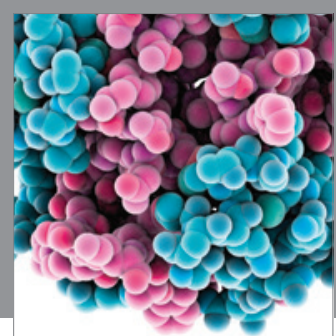

Journal of
Diabetes Research

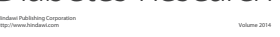

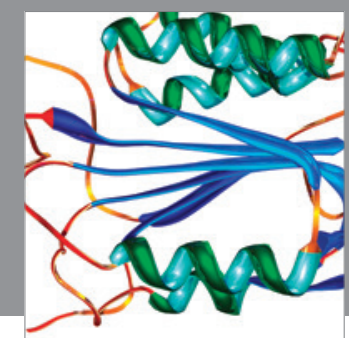

Disease Markers
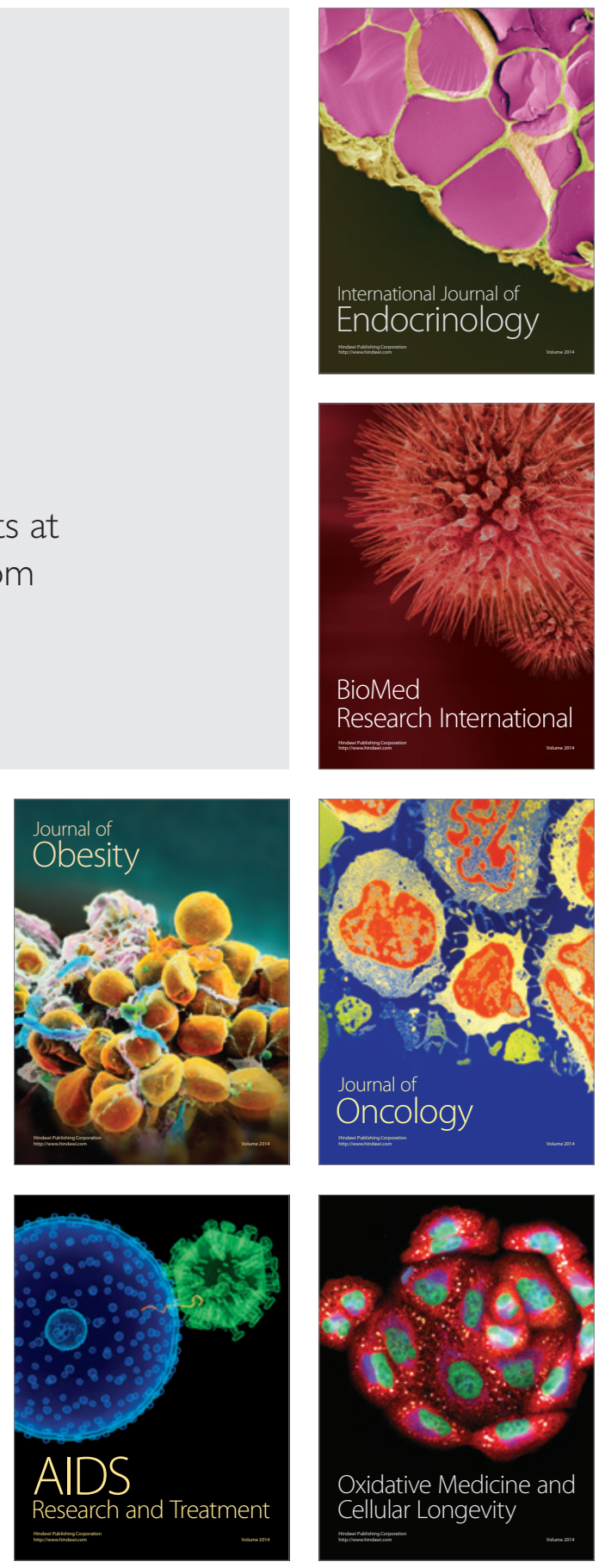